\title{
Hämophilie in der Orthopädie - Schwere Folgeschäden können vermieden werden
}

Schwere angeborene oder erworbene Gerinnungsstörungen wie Hämophilie $A$ oder B, von Willebrand-Syndrom und andere komplexere Faktorenmangelsyndrome führen häufig zu spontanen Muskel- und Gelenkblutungen sowie zum Teil zu lebensbedrohenden Einblutungen in die inneren Organe (ZNS, Nieren und andere). Auch bei Verletzungen und Operationen muß die gestörte Hämostase rechtzeitig diagnostiziert und therapiert werden.

Vor allem die rezidivierenden Gelenkeinblutungen im Kindesalter führen ohne eine adäquate Frühbehandlung innerhalb kurzer Zeit zu schweren Gelenkveränderungen. Durch die Resorption und den Abbau des freien Blutes aus der Gelenkhöhle kommt es zu Veränderungen der Synovialis und zu schweren sekundären Knorpelschäden. Diese hämophile Arthropathie mündet schließlich im Rahmen einer sekundären Arthrose in fortschreitenden Fehlstellungen und Gelenkversteifungen und führt zu einer schweren Behinderung der Patienten.

Durch eine konsequente Substitutionstherapie, die bereits im Kleinkindesalter begonnen werden sollte, lassen sich Spätschäden an den Gelenken vermeiden oder zumindest deutlich hinaus zögern. Auch bei erwachsenen Patienten kann durch eine Prophylaxe mit Gerinnungspräparaten die gestörte $\mathrm{Ge}$ lenkfunktion auch über einen langen Zeitraum erhalten werden. Die konservative und operative Behandlung von Blutern stellt somit eine interdisziplinäre Herausforderung dar und muß in enger Zusammenarbeit zwischen Hämostaseologen, Pädiatern, Orthopäden und anderen operativen Fachgebieten erfolgen.

Insbesondere bei operativen Maßnahmen ist die Kooperation mit einem Hämophilie-Zentrum notwendig, um eine optimale Diagnostik und perioperative Einstellung der Blutgerinnungsstörung zu gewährleisten. Auch die Rehabilitation von Hämophiliepatienten muß zwischen den einzelnen Fachgruppen abgestimmt werden.
Im vorliegenden Band wird eine aktuelle Übersicht zur Pathologie, Diagnostik und Therapie von Hämophiliepatienten in der Orthopädie gegeben. Neben der klassischen Hämophilie wird auch das von Willebrand-Syndrom dargestellt. Mit einer Inzidenz von ca. $1 \%$ in der Normalbevölkerung ist diese Hämostasestörung bei orthopädisch-traumatologischen Eingriffen zu beachten. Die Autoren verfügen jeweils über langjährige Erfahrungen in großen Hämophiliezentren.

\section{numenenr}

F. Kerschbaumer

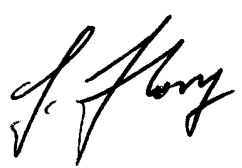

L. Hovy 